Cite this: Soft Matter, 2014, 10, 3649

\title{
Compact structure and non-Gaussian dynamics of ring polymer melts
}

\author{
Ana R. Brás, ${ }^{* a}$ Sebastian Gooßen, ${ }^{a}$ Margarita Krutyeva, ${ }^{a}$ Aurel Radulescu, ${ }^{\text {b }}$ \\ Bela Farago, ${ }^{c}$ Jürgen Allgaier, ${ }^{a}$ Wim Pyckhout-Hintzen, ${ }^{a}$ Andreas Wischnewski ${ }^{a}$ \\ and Dieter Richter ${ }^{\mathrm{a}}$
}

We present a neutron scattering analysis of the structure and dynamics of PEO polymer rings with a molecular weight 2.5 times higher than the entanglement mass. The melt structure was found to be more compact than a Gaussian model would suggest. With increasing time the center of mass (c.o.m.) diffusion undergoes a transition from sub-diffusive to diffusive behavior. The transition time agrees well with the decorrelation time predicted by a mode coupling approach. As a novel feature well pronounced non-Gaussian behavior of the c.o.m. diffusion was found that shows surprising analogies to the cage effect known from glassy systems. Finally, the longest wavelength Rouse modes are suppressed possibly as a consequence of an onset of lattice animal features as hypothesized in theoretical approaches.

Received 24th October 2013 Accepted 3rd February 2014

DOI: $10.1039 / c 3 s m 52717 d$

www.rsc.org/softmatter

While theoretical predictions and computer simulation

\section{Introduction}

The molecular rheology of polymer systems with their rich dynamics is one of the most fascinating fields of modern materials science. ${ }^{\mathbf{1 , 2}}$ The fundamental concepts that successfully describe the dynamics of polymers like the tube model including contour length fluctuations and constraint release processes or the hierarchical relaxation in branched systems - to name the most important ones - are heavily determined by the influence of chain ends. ${ }^{3}$ In the reptation model the topological confinement of long entangled polymer chains is represented by a virtual tube. ${ }^{4}$ The reptation process, i.e. the creep motion out of the tube, cannot exist without chain ends. ${ }^{3}$ Higher order processes like contour length fluctuations and constraint release relate to chain end fluctuations or the diffusive removal of a chain end section that is part of the confining tube.

Therefore one of the remaining important challenges in polymer science is summarized in one question: what happens when polymers have no ends at all? An elaborate study of the chain dynamics within a ring melt is a promising mission to address this fundamental aspect of polymer physics. Beyond that, rings are fundamental structures in biology. Mitochondrial and plasmic DNA are cyclic and often have a knotted structure; therefore ring polymers may serve as models for fundamental bio-physical problems as well.

ajülich Centre for Neutron Science JCNS (JCNS-1) and Institute for Complex Systems (ICS), Forschungszentrum Jülich GmbH, 52425 Jülich, Germany. E-mail: a.bras@ fz-juelich.de; Fax: +492461 61-2610; Tel: +49 2461 61-5093

${ }^{b} J$ ülich Centre for Neutron Science JCNS, Forschungszentrum Jülich GmbH, Outstation at FRM II, Lichtenbergstraße 1, 85747 Garching, Germany

'Institute Laue-Langevin (ILL), F-38042 Grenoble Cedex 9, France studies are available,$^{5-10}$ high quality experimental data on the microscopic structure and dynamics of rings are rare. One important reason for this deficiency is the impact of linear contaminants present in the samples. ${ }^{11}$ Only recently it has been possible to produce highly pure and monodisperse rings in quantities sufficient for elaborate studies. ${ }^{12}$

In this work we present a small angle neutron scattering (SANS) and neutron spin echo (NSE) study combined with pulsed-field-gradient (PFG)-NMR experiments on a $5 \mathrm{~kg} \mathrm{~mol}{ }^{-1}$ monodisperse and pure PEO ring (360 bonds per ring) melt. The following results stand out:

(i) The structural data demonstrate that the rings display a non-Gaussian compact structure. (ii) The chain center of mass motion (c.o.m.) crosses from a subdiffusive regime at short times to Fickian diffusion at longer times. The decorrelation time agrees with mode coupling predictions. (iii) In analogy to the cage escape in glassy dynamics, the c.o.m. diffusion displays non-Gaussian behavior, a phenomenon not observed before in polymer diffusion studies. (iv) Finally, long wavelength internal modes are suppressed, an observation that might indicate the onset of lattice animal features.

\section{Experimental}

\subsection{Samples}

Cyclic hydrogenous and deuterated polyethylene glycols were obtained from linear precursors under conditions of high dilution. ${ }^{13}$ Multiple fractionation allowed to remove byproducts of higher molecular weights completely and to reduce contamination by the linear mother compound. The chain ends of the linear residues were oxidized to carboxylic end groups, 
which then could be bound to an ion exchange resin whereas the non-oxidized cyclic product was eluted. This purification boosted the purity of the cyclic product to $>99.5 \%$ as was obtained from nuclear magnetic resonance $\left({ }^{1} \mathrm{H}-\mathrm{NMR}\right)$ for the hydrogenous material. The deuterated analog was synthesized and purified identically. Therefore the same quality must be assumed.

Size exclusion chromatography (SEC) in THF/N,N-dimethylacetamide (85/15) with PEG calibration yielded molecular weights $M_{\mathrm{n}}=5200 \mathrm{~g} \mathrm{~mol}^{-1}$ for the d-polymer and $M_{\mathrm{n}}=4980 \mathrm{~g}$ $\mathrm{mol}^{-1}$ for the h-polymer, respectively. From nuclear magnetic resonance $\left({ }^{1} \mathrm{H}-\mathrm{NMR}\right) M_{\mathrm{n}}=5470 \mathrm{~g} \mathrm{~mol}^{-1}$ for the d-polymer and $M_{\mathrm{n}}=5280 \mathrm{~g} \mathrm{~mol}^{-1}$ for the h-polymer were obtained. The polydispersity indices $M_{\mathrm{w}} / M_{\mathrm{n}}$ were 1.01 for the hydrogenous PEG and 1.03 for the deuterated PEG, respectively.

Blends of hydrogenous and deuterated polymers $(\mathrm{h} / \mathrm{d})$ were prepared in solution and freeze-dried from benzene. The volume fraction of the hydrogenous polymers in $\mathrm{h} / \mathrm{d}$ blends was $17 \%$ in all experiments.

\subsection{Neutron scattering}

SANS measurements were performed at the diffractometer KWS2@MLZ (Munich, Germany) in a range of scattering vectors $0.01 \AA^{-1}<Q<0.4 \AA^{-1}$, where $Q$ is defined as $(4 \pi / \lambda) \sin (\theta / 2)$, with $\theta$ the scattering angle and $\lambda$ the neutron wavelength. NSE studies on the same blends were performed at the spectrometer IN15 at the ILL (Grenoble, France). The wavelength of the incoming neutrons was chosen such that $Q$ values of $0.05,0.08$, $0.10,0.13$ and $0.20 \AA^{-1}$ at reasonable Fourier times were reached. At the lowest $Q$ values Fourier times up to 300 ns were accessed. To avoid residual crystallization, the temperature of the measurements was chosen to be $413 \mathrm{~K}$.

\subsection{Pulse-field-gradient NMR}

In order to determine the long-time (macroscopic) diffusion coefficient PFG-NMR measurements were performed on the same sample and at the same temperature as the NSE experiment.

The NMR measurements were carried out using a magnetic resonance analyser Bruker Minispec (mq20) that operates at an ${ }^{1} \mathrm{H}$ frequency of $20 \mathrm{MHz}$ and is equipped with a permanent magnet. Diffusion coefficients were measured using a standard stimulated echo pulsed-field-gradient sequence. ${ }^{14}$ The echo signal intensity is given by:

$$
A\left(q^{2}\right)=A(0) \exp \left(-q^{2}\left(\Delta-\frac{\delta}{3}\right) D\right)
$$

where, $q=\gamma \delta g, \gamma$ is the proton gyromagnetic ratio, $g$ and $\delta$ denote the amplitude and duration of the magnetic field gradient, respectively. The gradient pulse separation time $\Delta$ is defined as the observation time of the experiment and $\delta=5 \mathrm{~ms}$. The gradient amplitude $g$ was varied from 0 to $2.9 \mathrm{~T} \mathrm{~m}^{-1}$, leading to a decrease of the echo signal intensity by a factor of about 10 (Fig. 1).

The diffusion attenuation curves were found to be singleexponential, demonstrating a simple self-diffusion process to be expected for a monodisperse polymer. Additionally, no dependence on the observation time $\Delta$ was observed in the

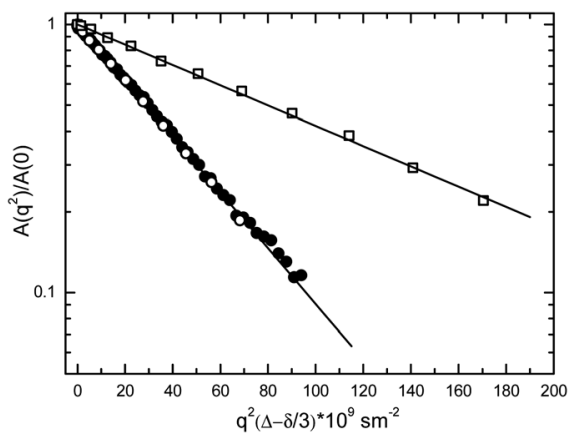

Fig. 1 Diffusion attenuation curves for ring (open circles) and linear (open squares) PEO measured at the observation time $\Delta=15 \mathrm{~ms}$. Filled circles represent the data for the ring PEO measured at $\Delta=100 \mathrm{~ms}$.

interval from 15 to $500 \mathrm{~ms}$. Thus the polymers undergo Fickian diffusion in the melt state (see Fig. 1). The diffusion coefficients were evaluated from the slope of the attenuation curves and were found to be $(2.44 \pm 0.34) \AA^{2} \mathrm{~ns}^{-1}$ for the ring PEO and $(0.87 \pm 0.07) \AA^{2} \mathrm{~ns}^{-1}$ for the equivalent linear chain.

\section{Neutron scattering results}

\subsection{Small angle neutron scattering}

Fig. 2a shows the Kratky representation of the obtained SANS scattering profiles for both the linear and the ring polymer, i.e. the second moment of the differential macroscopic scattering cross-section $\mathrm{d} \Sigma(Q) / \mathrm{d} \Omega Q^{2}$ in absolute units $\left(\AA^{-2} \mathrm{~cm}^{-1}\right)$, further abbreviated as intensity, $I(Q) Q^{2}$, versus scattering vector $Q$.

Assuming Gaussian statistics, the form factor for the ring polymer in the discrete representation reads

$$
P(Q)=\frac{1}{N^{2}}\left[N+2 N \sum_{k=1}^{N} F^{2 v} \exp \left\{-\frac{Q^{2} l^{2} k^{2}}{6} F^{2 v}\right\}\right]
$$

The finite length of the considered chains and its implication for the asymptotic $Q$ dependencies are herewith considered explicitly. The ring closure is taken into account by $F^{2 v}$ with $F^{2 \nu}=(1-k / N)^{2 \nu}, k$ being the segment number $(1<k<N), \nu$ the Flory excluded volume exponent and $l$ the segment length. Various suggestions for $F^{2 v}$ have been recently proposed. ${ }^{15}$ All approaches practically yield equally good descriptions of the data with variations in $\nu$ between 0.43 and 0.45 . This result already indicates a significant deviation from Gaussian statistics of these polymers in the bulk, for which $\nu=1 / 2$ is expected. We used this empirical modification to describe not only the SANS data but also the structural part of the Rouse model applied for the NSE data (vide infra). The linear chain is retrieved with $F=1$ and $\nu=1 / 2$.

To account for interchain contributions due to the small differences in the $\mathrm{h}$ - and d-chain lengths, together with eqn (2), we employed the random phase approximation, fixing thereby the chain lengths to the chemically determined values. A fit to the ring or linear chain SANS data led to radii of gyration $R_{\mathrm{G}, \mathrm{c}}=(15.64 \pm 0.08) \AA$ resp. $R_{\mathrm{G}, \mathrm{l}}=(24.58 \pm 0.24) \AA$. The linearto-ring ratio of 1.57 significantly exceeds the value of $\sqrt{2}$ that is 

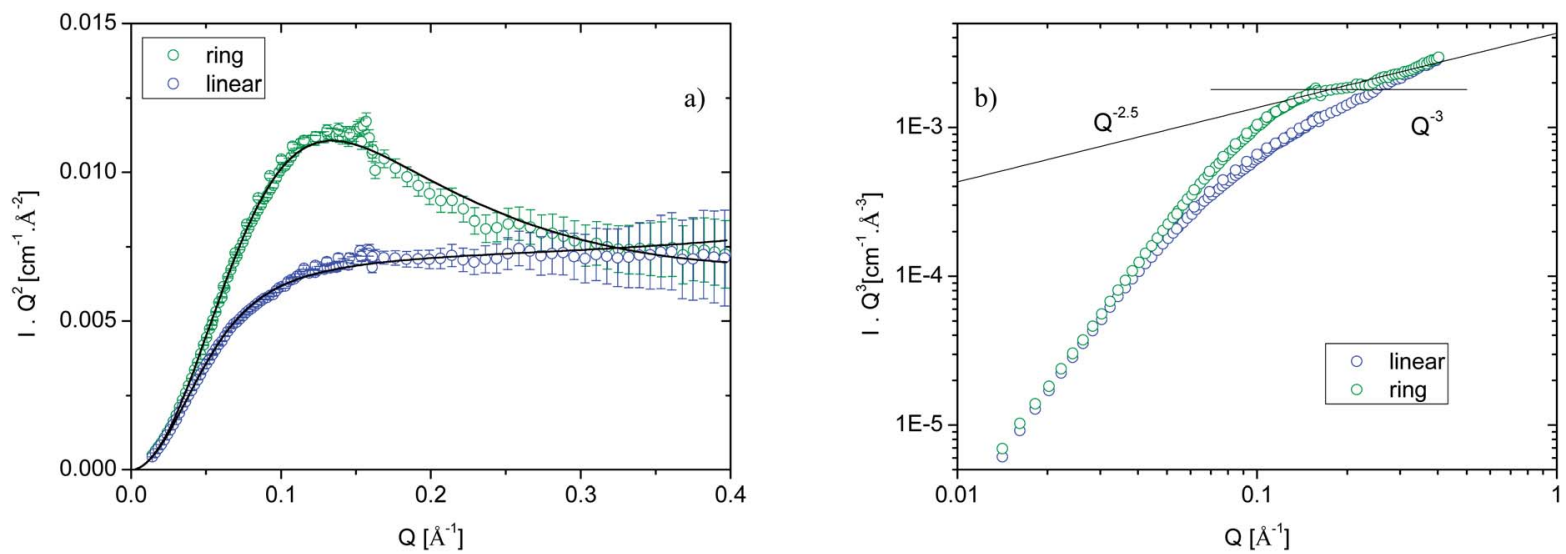

Fig. 2 (a) Kratky representation of the SANS data (symbols) and respective fits (lines) for the $5 \mathrm{~kg} \mathrm{~mol}^{-1} \mathrm{PEO}$ ring and its linear counterpart; (b) $/ Q^{3}$ representation of the SANS data for the $5 \mathrm{~kg} \mathrm{~mol}^{-1}$ PEO ring and its linear counterpart (the symbols are the same as in (a)).

predicted for Gaussian rings and signifies an increased compactness of the ring. In the intermediate $Q$-region the intensity decays with $S(Q) \propto Q^{-P}$ with $P=2.3$ leading to the peak observed in a $2^{\text {nd }}$ moment representation of the intensity (Fig. 2a). The best fit to eqn (2) required $\nu=(0.44 \pm 0.01)$, i.e. $P=1 / \nu=2.3$. The data in the intermediate $Q$ range satisfactorily agree within the statistical uncertainty with the presented model, although the experimental peak is slightly more pronounced than predicted by the model. This may be due to an even more enhanced compactness of the ring. This is in best agreement with recent computer simulations predicting a limiting behavior of $P=3$ (i.e. $\nu=1 / 3$ ) for high $N .{ }^{5}$ As shown in Fig. $2 \mathrm{~b}$ our data approach the predicted trend. According to ref. 5 , the investigated chain length is about one order of magnitude below the region where $\nu=1 / 3$ should be found and well above the Gaussian regime.

\subsection{Neutron spin-echo spectroscopy}

Fig. 3 presents NSE data for the ring and the linear chain observed at the lowest investigated $Q$-value $\left(Q=0.05 \AA^{-1}\right)$. The data represent the coherent dynamic structure factor of the ring and linear melts and to a very large extent they reflect the translational diffusion behavior of both polymers. Immediately

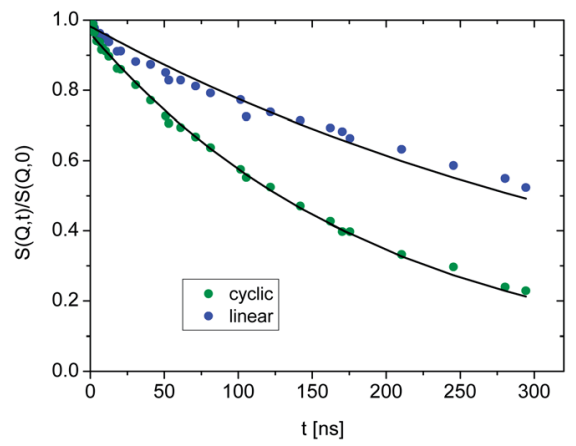

Fig. $3 S(Q, t) / S(Q, 0) v s$. $t$ for the ring and the linear chain at the lowest $Q$ value $\left(Q=0.05 \AA^{-1}\right)$. Error bars are smaller than the symbol size. The lines are a fit to the data with eqn (3). it is clear that the linear chain moves significantly more slowly than the ring - the decay of the structure factor is much weaker! In order to quantify the results we fitted the data with the Rouse equations for the linear chain ${ }^{16}$ and that modified for the ring topology. The Rouse mode spectrum predicted for cyclic polymers can be found in the literature. ${ }^{17}$ The solid lines in Fig. 3 show the result of the fit using solely the first part of the Rouse equation, $S(Q, t)=\exp \left(-Q^{2} D t\right)$, i.e. c.o.m. diffusion only.

$$
\begin{aligned}
S(Q, t)= & \frac{1}{N} \sum_{i, j} \exp \left[-Q^{2} D t-\frac{1}{6} Q^{2} l^{2}|i-j|^{2 \nu}\right. \\
& \times\left(1-\frac{|i-j|}{N}\right)^{2 v}-\frac{4 Q^{2} R_{\mathrm{g}}{ }^{2}}{\pi^{2}} \sum_{p=2, \mathrm{odd}} \frac{1}{p^{2}} \cos \left(p \pi \frac{(i-j)}{N}\right) \\
& \left.\times\left(1-\exp \left(\frac{-t p^{2}}{\tau_{\mathrm{R}}}\right)\right)\right]
\end{aligned}
$$

here, $i, j$ are the segment numbers and $\tau_{\mathrm{R}}$ is the Rouse time of the chain. The first part in the exponential describes the center of mass diffusion with the diffusion coefficient $D$, the second term represents the structure of the chain while the third part contains the segmental dynamics, represented by a sum over the Rouse modes $p$ of the polymer chain with a Rouse time, $\tau_{\mathrm{R}}=$ $\xi_{0} N^{2} l^{2} /\left(3 \pi^{2}\right) k_{\mathrm{B}} T, \xi_{0}$ being the monomeric friction coefficient. Essentially the spectrum differs from that of a linear chain by the absence of every second mode and a modification of the mode eigenvector. ${ }^{17}$ Since the center of mass diffusion is given by $D=\left(k_{\mathrm{B}} T\right) /(N \xi)$ and $l$ is determined from SANS, the Rouse analysis contains only one free parameter, namely the segmental friction parameter $\xi$.

The fitting procedure arrived at $D=(2.05 \pm 0.02) \AA^{2} \mathrm{~ns}^{-1}$ for the (more compact) pure rings, and $D=(0.94 \pm 0.01) \AA^{2} \mathrm{~ns}^{-1}$ for the linear chains. Note: especially in the case of the linear chain the description of the lowest $Q$ data shown by the solid lines is clearly not perfect - the dynamic structure factor appears to be more stretched than indicated by the theory.

There are two origins for this different behavior: (i) the chains have a molecular weight corresponding to about 2.5 times the entanglement distance. Therefore for the linear chain 
we expect the onset of topological constraints which will slow down the diffusion coefficient and modify the mode spectrum, while (ii) entanglements should not play a role for the ring polymers. Therefore deviations from the diffusion or internal dynamics as predicted by eqn (3) are observed. In the following we analyze in more detail the effects on the mode spectrum and its contribution to the structure factor.

In fact, the absence of every second mode has an important consequence: at the smallest investigated $Q=0.05 \AA^{-1}$ the ring $S(Q, t)$ is determined by c.o.m. diffusion only, which is not the case for the linear analog. To clarify this point, a mode contribution analysis using the unmodified or modified Rouse mode spectrum respectively was performed for both systems. The contribution of the different normal modes to the dynamic structure factor depends strongly on the momentum transfer $Q$. Since $S(Q, t)$ does not simply decompose into a sum of contributions from the different modes, the mode contribution factors were defined as ${ }^{18}$
The absence of mode contributions at low $Q$ for the ring allows to extract the c.o.m. mean square displacement (m.s.d.) directly from the measured $S(Q=0.05, t)$. Fig. 5 shows such obtained m.s.d.'s $v s$. Fourier times for $Q=0.05 \AA^{-1}$ from the NSE ring data. These can easily be extracted from the scattering function by $\left\langle r^{2}(t)\right\rangle=\left(-6 / Q^{2}\right) \ln \{S(Q, t)\}$. As found for melts of linear chains and as confirmed by computer simulations at short times the m.s.d. displays a sub-diffusive regime followed by a transition to Fickian diffusion $\left(\mathrm{t}^{1}\right)$ at longer times. ${ }^{19}$ As presented in ref. 20 this subdiffusive behaviour can be understood in terms of an inter-chain potential. The influence of this potential becomes negligible as soon as the polymer has travelled a distance comparable to its radius of gyration. This model leads to a decorrelation time by $\tau_{\text {decorr }}={R_{\mathrm{G}}}^{2} / D$. Using the $R_{\mathrm{G}}$ as determined by SANS and the "true diffusion" as extracted from the Fickian regime in Fig. 5, we find $\tau_{\text {decorr }}=125 \mathrm{~ns}$, in very good agreement with the experiment.

The diffusion coefficient as measured by NSE and by PFGNMR is shown in Fig. 5 as lines. They correspond to the mean squared displacement constructed as, $\left\langle r^{2}(t)\right\rangle=6 D t$, where $D$ is

$$
R_{\mathrm{p}}(Q)=\sum_{i, j} \frac{\exp \left\{-\frac{1}{6} Q^{2} l^{2}|i-j|^{2 v}\left(1-\frac{|i-j|}{N}\right)^{2 v}-\frac{4 R_{\mathrm{G}}^{2} Q^{2}}{\pi^{2}} \frac{1}{p^{2}} \cos \left(p \pi \frac{(i-j)}{N}\right)\right\}}{S(Q, t=0)}
$$

$R_{\mathrm{p}}(Q)$ describes to what extent a mode " $p$ " may relax $S(Q, t)$ in the limit of long times $(t \rightarrow \infty)$ and under the premise that all other modes are not active. Fig. 4 displays these mode contribution factors for the linear $(p=1$ and $p=2)$ and for the ring chains $(p=2$ and $p=4)$. In the linear case it is obvious that segmental relaxation already leads to a decay of $S(Q, t)$ in the entire $Q$ and time range probed by the NSE experiment. In contrast to the linear analog, for ring polymers we expect only translational diffusion contributions except for $Q$ values higher than $0.1 \AA^{-1}$. Therefore the faster c.o.m. diffusion of the rings compared to the linear analog, as well as the problem with the fit of the latter at the lowest $Q$ (see Fig. 3), is obviously only due to the onset of entanglements in the case of the linear polymer.

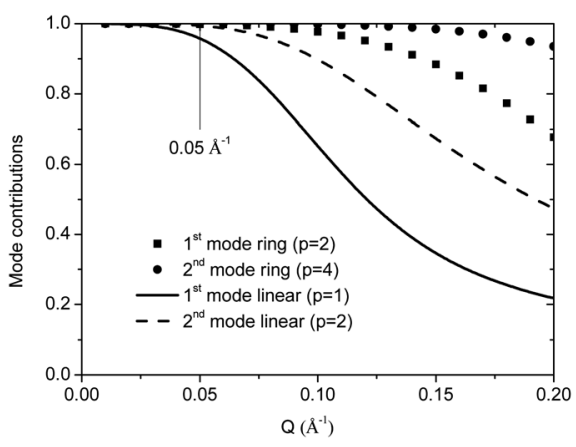

Fig. 4 Contribution factors for the first modes of both the ring and the linear chains to $S(Q, t) / S(Q, 0)$ (see legend in the figure). Note that the first mode for the rings is $p=2$. the diffusion coefficient obtained by NSE and PFG-NMR, respectively. The PFG-NMR value is in very good agreement with the NSE result.

\section{Discussion}

The observed sub-diffusive c.o.m. motion in Fig. 5 is not considered in the Rouse model. While this has generally been neglected in the past, the theory can easily be adapted by

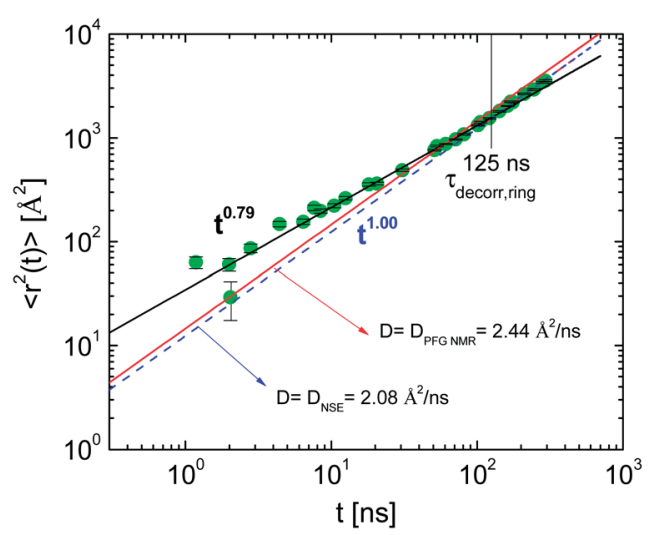

Fig. 5 C.o.m. m.s.d. extracted from the $Q=0.05 \AA^{-1}$ NSE data for PEO rings. The error bars have been calculated using the theoretical general formula for error propagation. For times shorter than $125 \mathrm{~ns}$ sub-diffusive c.o.m. is observed. For longer times the c.o.m. diffusion changes to Fickian diffusion. The blue and red lines represent the diffusion as measured by NSE and by PFG-NMR, respectively. Both values are in excellent agreement. The black line shows a $t^{0.79}$ power law. 
replacing the term for the c.o.m. diffusion $\mathrm{e}^{-Q^{2} D t}$ by $\mathrm{e}^{\left[-r^{2}(t) Q^{2} / 6\right]}$ with $r^{2}(t)$ describing the experimental data.

With this approach, i.e. by just considering the Gaussian form $\mathrm{e}^{\left[-r^{2}(t) Q^{2} / 6\right]}$ we now calculate the diffusive contribution to $S(Q, t)$ for all $Q$. We obtain the dashed lines in Fig. 6 .

Now, $S(Q, t)$ at $Q=0.05 \AA^{-1}$ naturally is described perfectly. However, solely based on c.o.m. diffusion the relaxation at intermediate $Q$ is already over-predicted. This over-prediction demonstrates that the Gaussian approximation, i.e. the $Q^{2}$ dependence of the diffusion relaxation function, appears to be violated - the c.o.m. diffusion displays non-Gaussian behavior. Note that this non-Gaussian c.o.m. diffusion has already been discussed for linear melts. ${ }^{21,22}$ On the other hand for $Q=0.2 \AA^{-1}$ the calculated decay under-predicts the experimental data here obviously segmental relaxation contributes to $S(Q, t)$.

Correcting for the Gaussian approximation of $S(Q, t)$ to a first order the non-Gaussian effects displayed in Fig. 6 may be described in terms of a positive fourth order term in $Q::^{23,24}$

$$
S(Q, t)=\exp \left(\frac{-\left\{r^{2}(t)\right\} Q^{2}}{6}+\frac{\alpha(t) Q^{4}\left\{r^{2}(t)\right\}^{2}}{72}\right)
$$

here $\alpha(t)$ is the time dependent non-Gaussianity parameter. To extract $\alpha(t)$ from the NSE data, the apparent m.s.d., i.e. the logarithm of the above formula divided by $Q^{2} / 6$ is plotted $v s . Q^{2}$ for each Fourier time. These points can be fitted by a linear function, where the intersection of this line with the $y$-axis represents the "true" m.s.d., while the slope of this line allows to extract $\alpha(t)$ for this particular Fourier time. For the $5 \mathrm{~kg} \mathrm{~mol}^{-1}$ PEO ring melt this procedure had to be restricted to the two lowest $Q$, because as shown in Fig. 4 exclusively in this $Q$ range no possible mode contribution exists. With the obtained $\alpha(t)$ the correction towards the 'true' $\left\langle r^{2}(t)\right\rangle$ are found to be negligible. Therefore, in a second step we used the analytic interpolation formula for $\left\langle r^{2}(t)\right\rangle,\left\langle r^{2}(t)\right\rangle=\left(37.842 t^{0.79081}(1 /(1+\exp ((x-125) /\right.$ $131.124)))+12.278 t(1-(1 /(1+\exp ((x-125) / 131.124)))))$ in order to extract $\alpha(t)$ from eqn (5) for $Q=0.08 \AA^{-1}$.

The data points in Fig. $7 \mathrm{~b}$ show the result of this procedure. In particular at longer times $\alpha(t)$ is low, however at intermediate

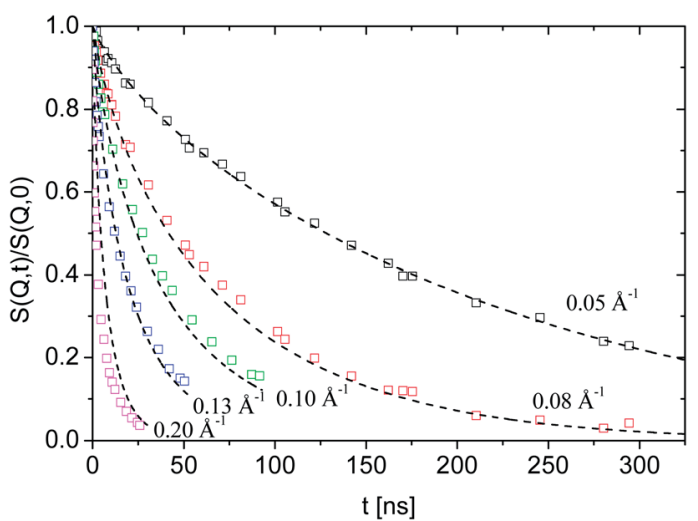

Fig. 6 Dynamic structure factor for the $5 \mathrm{~kg} \mathrm{~mol}^{-1} \mathrm{PEO}$ ring melt for different $Q$-values. Error bars for all $Q$-values are smaller than the symbol size. The dotted black lines show the c.o.m. diffusion including the subdiffusive behaviour (see text).
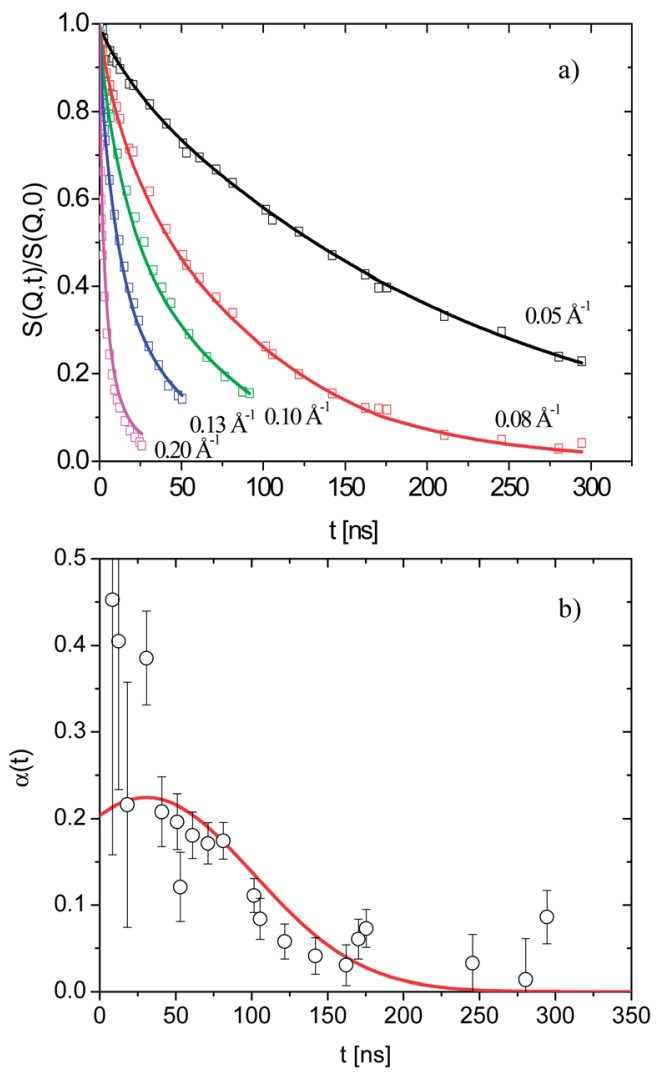

Fig. 7 Dynamic structure factor for the $5 \mathrm{~kg} \mathrm{~mol}^{-1}$ PEO ring melt at different $Q$-values. (a) Coloured lines depict the Rouse model fit taking into account the subdiffusivity and including the non-Gaussianity parameter function $\alpha(t)$. Long Rouse modes are suppressed (see text). (b) The plot shows the non-Gaussianity parameter $\alpha(t)$ from eqn (5) (see text) compared with the $\alpha(t)$ obtained from the fit to the $S(Q, t)$ data (red full line)

times values of 0.2 to 0.3 are reached, a clear indication of nonGaussian center of mass diffusion of the rings.

Finally, an alternative way of extracting $\alpha(t)$ from the $S(Q, t)$ data has been taken. All $S(Q, t)$ data were fitted with a Gaussian shape for $\alpha(t)$ (see later) additionally allowing for mode contributions. However, as already may be suspected from Fig. 4 and other than predicted by theory the fitting procedure evidences that the longer wavelength Rouse modes do not seem to relax the rings. To take this into account we limited the contributing modes by a minimum value $p_{\min }$ that removed long wavelength modes with $p<p_{\text {min }}$. The result is shown in Fig. 7a. Now the fit describes all data very well, the obtained $\alpha(t)$ compares reasonably well with the values determined for each single time separately (see Fig. $7 \mathrm{~b}$ ). The resulting $p_{\min }=4$ reveals that the first allowed Rouse mode $(p=2)$ for ring topology is suppressed and only modes with wavelengths shorter than half the ring size are allowed. It should be mentioned that allowing mode $p=2$ to contribute cannot be compensated by different $\alpha(t)$, i.e. the obtained combination of $\alpha(t)$ and $p_{\min }$ is the only possible way to get a good description of the $S(Q, t)$ data. While for a linear chain the first mode exhibits one knot or the wavelength is twice the full chain length, for the first ring active mode the amplitude pattern is characterized by 4 knots. Lower Fourier 
components are absent. This pattern, connected with the closed shape of the ring polymer, might be a first indication of a lattice animal formation.

For a better quantitative determination of non-Gaussian behavior, $S(Q, t)$ data over a wider $Q$-range that are solely influenced by c.o.m. diffusion are needed. This implies - at least if the single chain dynamic structure factor is concerned - shorter chains. An approach to lower $Q$ would not help, since then the $Q^{4}$ term in eqn (5) becomes negligible. In ref. 12 we investigated $2 \mathrm{~kg} \mathrm{~mol}{ }^{-1}$ PEO rings. Since the NSE data for that sample were not influenced by segmental modes at all, these small rings are ideal candidates to verify the existence and to explore the shape of the time dependent non-Gaussianity parameter for the c.o.m. diffusion of polymer rings. $\alpha(t)$ values for the $2 \mathrm{~kg} \mathrm{~mol}^{-1}$ rings are obtained by the same procedure as applied initially to the large rings. The result is displayed in Fig. 8. Note, that here $S(Q, t)$ data from five different $Q$ values were used to determine $\alpha(t)$ over the entire time range. In spite of the still modest statistics we may draw two conclusions from this result: (i) the non-Gaussianity parameter with values between 0.1 and 0.25 is not negligible (typical values in glasses are around 0.4) corroborating the observations made with the $5 \mathrm{~kg} \mathrm{~mol}^{-1}$ sample and (ii) the peak structure in the $\alpha(t)$ is evident, a phenomenon that has not been observed before for the c.o.m. diffusion in a polymer melt. Note that typical values for the non-Gaussianity parameter in glasses are 0.4 and it was found for the $\alpha$-relaxation that values below 0.2 are negligible. ${ }^{23,24}$ This is obviously not the case for the c.o.m. diffusion on polymer ring melts.

Going back to original ideas of non-Gaussianity and to earlier investigations mainly on glassy systems, Fig. 8 reminds on what was found for molecular glasses and referred to as the "cage effect". ${ }^{21,24}$ In a glass forming system one molecule is captured in a cage built by the adjacent molecules. There it rattles around, testing possible escape routes from the cage. This leads to an increase of $\alpha(t) .{ }^{24}$ When the molecule eventually escapes the cage confinement the non-Gaussianity parameter goes through a maximum, and for longer times decreases again. Translating this picture to our polymer ring system and having in mind that these rings are very compact objects, the peak time $\tau_{\text {peak }}$ of $\alpha(t)$ would refer to an escape time of a ring from the cage formed by the adjacent rings. In fact $\tau_{\text {peak }}$ is roughly $12 \mathrm{~ns}$ which is in a surprising agreement with $\tau_{\text {decorr }}=9 \mathrm{~ns}$ published in ref. 12. As mentioned above $\tau_{\text {decorr }}$ marks the time at which the

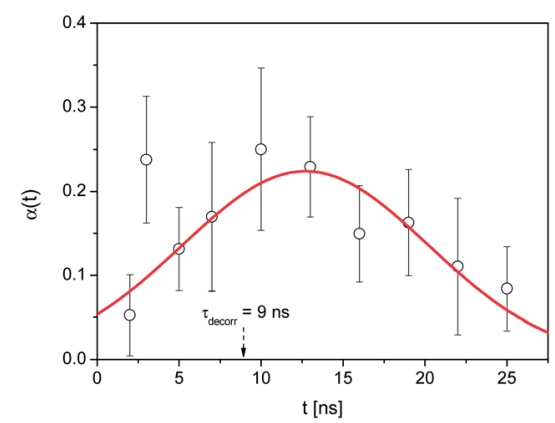

Fig. 8 Non-Gaussianity parameter $\alpha(t)$ for PEO rings with $M_{\mathrm{w}}=2 \mathrm{~kg}$ $\mathrm{mol}^{-1}$. The line is a guide for the eye. polymer chain has escaped from an inter-chain potential by traveling roughly the distance of its own size $\left(R_{\mathrm{G}}\right)$. For the larger ring $\alpha_{\max }$ is found at about $30 \mathrm{~ns}$, a factor of 4 smaller than $\tau_{\text {decorr }}=125 \mathrm{~ns}$. Seemingly the cage effect is now restricted to portions of the ring, possibly to the pattern indicated by the internal mode structure.

\section{Conclusions}

In summary neutron scattering and PFG-NMR experiments on only recently available highly monodisperse and pure ring polymers have unraveled novel static and dynamic properties. We have found a compact structure of the $5 \mathrm{~kg} \mathrm{~mol}^{-1}$ ring yielding a radius of gyration by a factor of 1.57 smaller compared to its linear counterpart. Since theoretical calculations for a cyclic Gaussian polymer predict a factor of $\sqrt{2}$, our result points to a more pronounced compactness. At intermediate $Q$, a slope of -2.3 , was found agreeing with trends from recent computer simulations that predict a slope of -3 in the limit of high $N .{ }^{5}$ The low $Q$ NSE data reveal sub-diffusive c.o.m. diffusion for times below $125 \mathrm{~ns}$. The transition to Fickian diffusion agrees well with mode coupling considerations and the long-time diffusion extracted from the NSE data is confirmed by independent PFGNMR experiments. Implementing this sub-diffusivity in the description of all NSE data, the Rouse approach fails, even the c.o.m. diffusion turns out to be non-Gaussian. Taking this into account allows the internal dynamics to be determined. It turns out that long wavelength Rouse mode is suppressed, a possible indication of a lattice animal formation. While an accurate evaluation of the non-Gaussianity parameter $\alpha(t)$ for the larger rings is difficult due to the limited number of $Q$-values that are not contaminated by internal modes, for $\alpha(t)$ of a small $2 \mathrm{~kg}$ $\mathrm{mol}^{-1}$ ring a systematic time dependence is observed. The peak in $\alpha(t)$ is evocative of the cage effect, as is known from molecular glasses. This comparison is substantiated by inspecting the peak position of $\alpha(t)$ : obviously the polymer ring has to travel its own $R_{\mathrm{G}}$ before it can escape from the interchain-potential which may be identified with the cage as is known from glasses.

\section{Acknowledgements}

The authors acknowledge EU for funding through the ITN214627 (DYNACOP) and through FP7 by means of the grant agreement for the Integrated Infrastructure Initiative N. 262348 ESMI. ARB and MK acknowledge DFG-SPP1568 and DFGSPP1369, respectively for financial support.

\section{Notes and references}

1 T. McLeish, Science, 2002, 297, 2005.

2 T. McLeish, Nat. Mater., 2008, 7, 933.

3 M. Doi and S. F. Edwards, The Theory of Polymer Dynamics, Clarendon, Oxford, 1986.

4 P. G. De Gennes, J. Chem. Phys., 1971, 55, 572.

5 J. D. Halverson, G. S. Grest, A. Y. Grosberg and K. Kremer, Phys. Rev. Lett., 2012, 108, 038301. 
6 J. D. Halverson, W. B. Lee, G. S. Grest, A. Y. Grosberg and K. Kremer, J. Chem. Phys., 2011, 134, 204904.

7 J. D. Halverson, W. B. Lee, G. S. Grest, A. Y. Grosberg and K. Kremer, J. Chem. Phys., 2011, 134, 204905.

8 T. Vettorel, A. Y. Grosberg and K. Kremer, Phys. Biol., 2009, 6, 025013.

9 S. Y. Reigh and D. Y. Yoon, ACS Macro Lett., 2013, 2, 296.

10 K. Hur, C. Jeong, R. G. Winkler, N. Lacevic, R. H. Gee and D. Y. Yoon, Macromolecules, 2011, 44, 2311.

11 M. Kapnistos, M. Lang, D. Vlassopoulos, W. PyckhoutHintzen, D. Richter, D. Cho, T. Chang and M. Rubinstein, Nat. Mater., 2008, 7, 997.

12 A. R. Brás, R. Pasquino, T. Koukoulas, G. Tsolou, O. Holderer, A. Radulescu, J. Allgaier, V. G. Mavrantzas, W. Pyckhout-Hintzen, A. Wischnewski, D. Vlassopoulos and D. Richter, Soft Matter, 2011, 7, 11169.

13 T. S. Sun, G. R. Yu, C. Price, J. Cooke and A. J. Ryan, Polymer, 1995, 36, 3775.

14 E. O. Stejskal and J. E. Tanner, J. Chem. Phys., 1965, 42, 288.
15 A. Bensafi, U. Maschke and M. Benmouna, Polym. Int., 2000, 49, 175183.

16 P. E. Rouse, J. Chem. Phys., 1953, 21, 1272.

17 G. Tsolou, N. Stratikis, C. Baig, P. S. Stephanou and V. G. Mavrantzas, Macromolecules, 2010, 43, 10692.

18 D. Richter, L. Willner, A. Zirkel, B. Farago, L. J. Fetters and J. S. Huang, Phys. Rev. Lett., 1993, 71, 4158.

19 W. Paul, G. D. Smith and D. Y. Yoon, Macromolecules, 1997, 30, 7772 .

20 M. Zamponi, A. Wischnewski, M. Monkenbusch, L. Willner, D. Richter, P. Falus, B. Farago and M. G. Guenza, J. Phys. Chem. B, 2008, 112, 16220.

21 W. Paul and G. D. Smith, Rep. Prog. Phys., 2004, 67, 1117.

22 G. D. Smith, W. Paul, M. Monkenbusch and D. Richter, J. Chem. Phys., 2001, 114, 4285.

23 R. Zorn, Phys. Rev. B: Condens. Matter Mater. Phys., 1997, 55, 6249.

24 A. Arbe, J. Colmenero, F. Alvarez, M. Monkenbusch, D. Richter, B. Farago and B. Frick, Phys. Rev. Lett., 2002, 89, 245701. 